\title{
Role of Lactobacillus in cervical cancer
}

This article was published in the following Dove Press journal:

Cancer Management and Research

\author{
Xi Yang' \\ Miao $\mathrm{Da}^{2}$ \\ Wenyuan Zhang ${ }^{3}$ \\ Quan $\mathrm{Qi}^{4}$ \\ Chun Zhang ${ }^{5}$ \\ Shuwen $\mathrm{Han}^{4}$ \\ 'Department of Intervention and \\ Radiotherapy, Huzhou Central \\ Hospital, ${ }^{2}$ Medical College of Nursing, \\ Huzhou University, ${ }^{3}$ Department of \\ Gynaecology, ${ }^{4}$ Department of Medical \\ Oncology, ${ }^{5}$ Department of Infectious \\ Diseases, Huzhou Central Hospital, \\ Huzhou, Zhejiang Province, People's \\ Republic of China
}

Correspondence: Shuwen Han Department of Medical Oncology, Huzhou Central Hospital, No 198 Hongqi Road, Huzhou, Zhejiang Province 313000 , People's Republic of China

Tel +86572 2023301

Email shuwenhan985@I63.com

\begin{abstract}
Cervical cancer is a common malignant cancer among women worldwide. Changes in the vaginal microecological environment lead to multiple gynecological diseases, including cervical cancer. Recent research has shown that Lactobacillus may play an important role in the occurrence and development of cervical cancer. This review explores the role of Lactobacillus in cervical cancer. A total of 29 articles were included after identification and screening. The pertinent literature on Lactobacillus in cervical cancer from two perspectives, including clinical studies and experimental studies, was analyzed. An association network for the mechanism by which Lactobacillus induces cervical cancer was constructed. In addition, we provide direction and insight for further research on the role of Lactobacillus in cervical cancer.
\end{abstract}

Keywords: CIN, cervical cancer, Lactobacillus, microorganism

\section{Introduction}

Cervical cancer is the third most commonly diagnosed cancer and the fourth leading cause of cancer-related deaths among women worldwide. ${ }^{1,2}$ Epidemiological investigations have shown that the number of new cases of cervical cancer per year is $\sim 485,000$, with 236,000 deaths occurring per year globally. ${ }^{3}$ Patients with cervical cancer often exhibit no obvious symptoms and signs at early stages; thus, the disease tends to be ignored and is easily misdiagnosed. Cervical cytologic screening has generally been used to detect cervical cancer and premalignant lesions over the past decade, and early diagnosis and appropriate treatment reduce the risk of cancer-related death. ${ }^{4}$

Current research has shown that microorganisms may play an important role in the occurrence and development of cervical cancer. ${ }^{5}$ Persistent infection with high-risk human papillomavirus (HR-HPV) is now believed to be a major causal factor in the development of the disease. ${ }^{6}$ The bacteria in the vagina maintain a dynamic balance under physiological conditions, but the imbalance of vaginal flora leads to multiple gynecological diseases, such as coleitis, high-grade cervical intraepithelial neoplasia (CIN), and cervical cancer. ${ }^{7}$ It has been confirmed that Gardnerella and Monilia are the main bacteria that induce coleitis. ${ }^{8,9}$ Previous research has shown that the abundance of vaginal flora such as Mycoplasma genitalium, ${ }^{10}$ aerobic lactobacilli, Staphylococcus epidermidis, Enterococci, Escherichia coli, and Bacteriodes species ${ }^{11}$ in patients with cervical cancer is different from that in healthy controls. However, the relationship between vaginal flora and cervical cancer has not yet been elucidated.

Lactobacillus is a group of bacteria that can act as a catalyst to produce lactic acid during the process of glycolysis. ${ }^{12}$ This group is the predominant bacteria in the healthy vagina and plays an important role in the protection of the female reproduction 
system. ${ }^{13}$ Summarizing previous studies, Lactobacillus in the vagina exerts its protective functions mainly through the following four potential mechanisms: 1) By preventing pathogenic bacteria from adhering to the epithelial tissue: vaginal epithelial cells (VECs) of fertile woman encounter periodic changes including hyperplasia, peeling, and repair under the effect of estrogen and progestin. Free glycogen that is produced during this process supplies matter and energy for the growth of Lactobacillus. Lactobacillus is adsorbed and occupies the VECs, and these bacteria can prevent the conglutination of invasive pathogenic bacteria that induce malignant tumors. ${ }^{14-16}$ 2) By secreting organic acid: Lactobacillus produces organic acid by decomposing glucogen to maintain the vaginal acidic environment, ${ }^{17}$ which can inhibit the growth and resist the invasion of pathogenic bacteria. In addition, the vaginal acidic environment is beneficial to maintain the activity of bacteriocins and $\mathrm{H}_{2} \mathrm{O}_{2} \cdot{ }^{18}$ 3) By secreting various metabolites: exopolysaccharides (EPSs), phosphorylated polysaccharides, and peptidoglycans, which are secreted by Lactobacillus, can inhibit the proliferation of malignant tumors. ${ }^{19,20}$ Bacteriocin and surface-active components can inhibit the production of tumorigenic substances and the growth of harmful microorganisms. ${ }^{21} \mathrm{H}_{2} \mathrm{O}_{2}$, which is also secreted by Lactobacillus, can directly kill harmful microorganisms or act in a bactericidal manner though the peroxidase-hydrogen peroxide-halide bactericidal system. ${ }^{18}$ 4) By activating the immune system: Lactobacillus affects cellular and humoral immunity. On one hand, these bacteria can increase the proliferation and differentiation of thymusderived cells (T cells). ${ }^{22}$ On the other hand, Lactobacillus, as an immune sensitizer, can increase immunological recognition and proliferation of bone marrow-derived cell (B cells). ${ }^{23}$ Lactobacillus also produces nitric oxide (NO) by stimulating macrophages and disrupting the energy metabolism of cancer cells. ${ }^{24}$ Considering the importance of lactobacilli in cervical cancer, in this review, we comprehensively analyzed and classified the pertinent literature on lactobacilli in cervical cancer from two perspectives, clinical case investigations, and studies of molecular mechanisms. We also provide direction and insight for further research on intestinal flora in cervical cancer.

\section{Methods}

The databases PubMed, Embase, and Cochrane were searched for literature published up to January 10, 2018. To achieve maximum sensitivity of the search strategy and identify all studies, the following terms were combined: ("cervix uterus" or "neck of uterus" or "uterine neck" or "cervical" or "cervix" or "uterine cervical" or "uterine cervix") and ("neoplasms" or "tumor" or "carcinoma" or "cancer" or "intraepithelial neoplasia" or "intraepithelial neoplasms") and ("lactic acid bacteria" or "lactobacillus" or "lactobacilli" or "vagina/ vaginal flora" or "vagina/vaginal microflora" or "vagina/ vaginal microorganism" or "vagina/vaginal microbiome" or "vagina/vaginal microbiota" or "vagina/vaginal microbe" or "vagina/vaginal microbiology" or "vagina/vaginal bacteria" or "vagina/vaginal bacterium"). All relevant abstracts were independently retrieved by two authors, and the articles with available information for the present systematic review were fully reviewed. A total of 29 articles were included after identification and screening. The detailed search strategy is presented in Figure 1.

\section{Study selection}

Studies adhering to the following criteria were considered for inclusion: 1) those published in English and 2) those involving Lactobacillus in CIN or cervical cancer in vivo or in vitro. The exclusion criteria were as follows: 1) letters, case reports, reviews, or conference reports; 2) the main studies not focused on the topic of vaginal Lactobacillus in CIN or cervical cancer; and 3) correlation clinical studies with a low quality score according to the Grading of Recommendations, Assessment, Development and Evaluation (GRADE) method. ${ }^{25}$

\section{Results}

CIN, which is considered to be a precancerous lesion of the cervix, ${ }^{26}$ was included in the present study. After identification, screening, and validation, we identified a total of 29 pertinent studies related to Lactobacillus and several of its subgenera in cervical cancer and precancerous lesions of the cervix published in recent years. The 29 pertinent studies were divided into two groups that comprised 14 clinical studies in one group and 15 experimental studies in the other group. The clinical studies and experimental studies are shown in Tables 1 and 2, respectively.

As shown in Table 1, 16S rRNA gene sequencing was the main method used to detect microbial community structure and relative abundance. The presence of cervical cancer and precancerous lesions in women was associated with a high relative abundance of Lactobacillus iners and Lactobacillus sp and low relative abundance of Lactobacillus jensenii and Lactobacillus crispatus. Two investigations revealed that the abundance of Lactobacillus in women with human papillomavirus (HPV) infection was lower (panel numbers 6 and 10). A randomized, double-blind, placebo-controlled study 


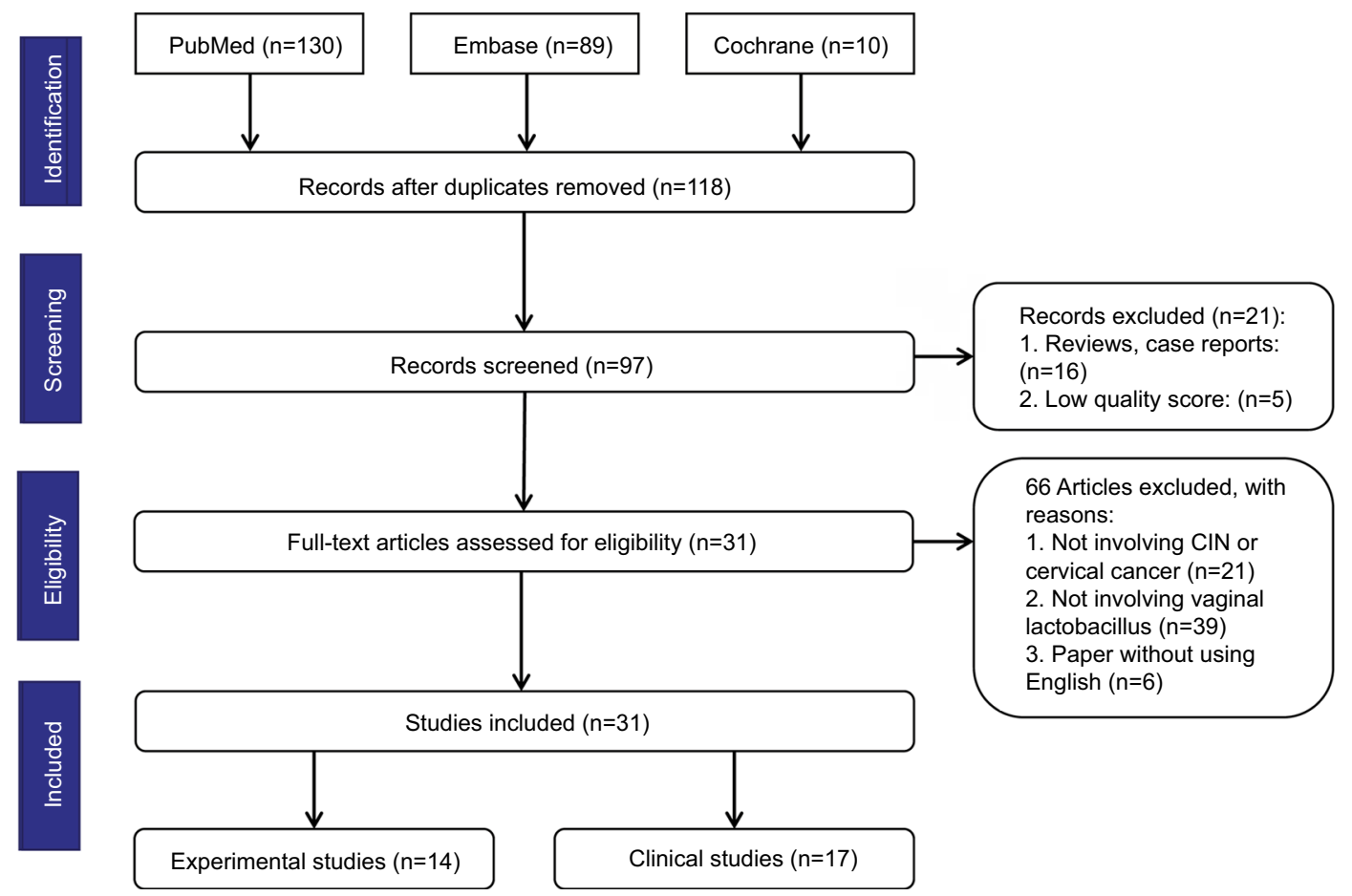

Figure I Literature search strategy.

Notes: Databases including PubMed, Embase, and Cochrane were searched for literature published up to January 10, 2018. A total of 29 studies including 17 clinical studies and 14 experimental studies were included after identification and screening. Abbreviation: CIN, cervical intraepithelial neoplasia.

showed that an oral lactobacilli (panel number 11) and an HR-HPV E7-expressing Lactobacillus-based vaccine (panel number 8 ) were able to decrease the risk of CIN. Moreover, two randomized controlled trial studies (panel numbers 12 and 13) supported the hypothesis that LC9018 (a biological response modifier prepared from heat-killed Lactobacillus casei YTT9018) was able to protect patients from radiationinduced leukopenia with few side effects.

As shown in Table 2, cancer cell and animal models were used along with molecular biology techniques such as the cell counting kit-8 assay, Western blots, immunohistochemistry, polymerase chain reaction, and enzyme-linked immunosorbent assays to study the role of Lactobacillus and several subgenera in cervical cancer. The results showed that Lactobacillus, its subgenera, and their supernatants exhibited antimetastatic and antiproliferative activities in cervical cancer cell lines by regulating cancer-related genes and eliciting an immunological response. Lactobacillus inhibited the viability of cervical cancer cells through regulating HPV oncogenes (panel numbers 1 and 6), and HPV-type $16 \mathrm{E} 7$ protein displayed on lactobacillus could protect against HPV-induced tumors through regulating cellular immunity (panel numbers 10-14). In particular, a negative result showed that $L$. casei extract was not able to inhibit the viability of cervical cancer cells in vitro (panel number 7).
As shown in Figure 2, a network of the mechanisms of Lactobacillus in cervical cancer was constructed to better visualize the theory behind the experimental studies.

\section{Discussion}

The vaginal microecological balance is dynamic and relative, and patients are able to recover from slight vaginal dysbacteriosis. The persistence of vaginal dysbacteriosis is thought to promote gynecological cancer. ${ }^{7,27}$ A decline in the quantity and activity of Lactobacillus leads to an overgrowth of anaerobic bacteria. ${ }^{28}$ Deleterious metabolites such as nitrous acid can be produced by these organisms, and the risk of HPV infection also increases. ${ }^{29}$ Persistent infection of oncogenic HPV is a cause of cervical cancer. ${ }^{6}$ The abundance of Lactobacillus in HPV infection is lower, and HPV oncogenes may be involved in regulation of the viability of cervical cancer cells inhibited by Lactobacillus. CIN, precancerous lesions of the cervix, is much more likely to occur with dysbacteriosis. Thus, the regulation and control of Lactobacillus may block the progression of cervical cancer.

The present study attempts to clarify the cross-talk between Lactobacillus and cervical cancer using both clinical and experimental studies by reviewing the pertinent literature. Experimental study can lay an important foundation for 


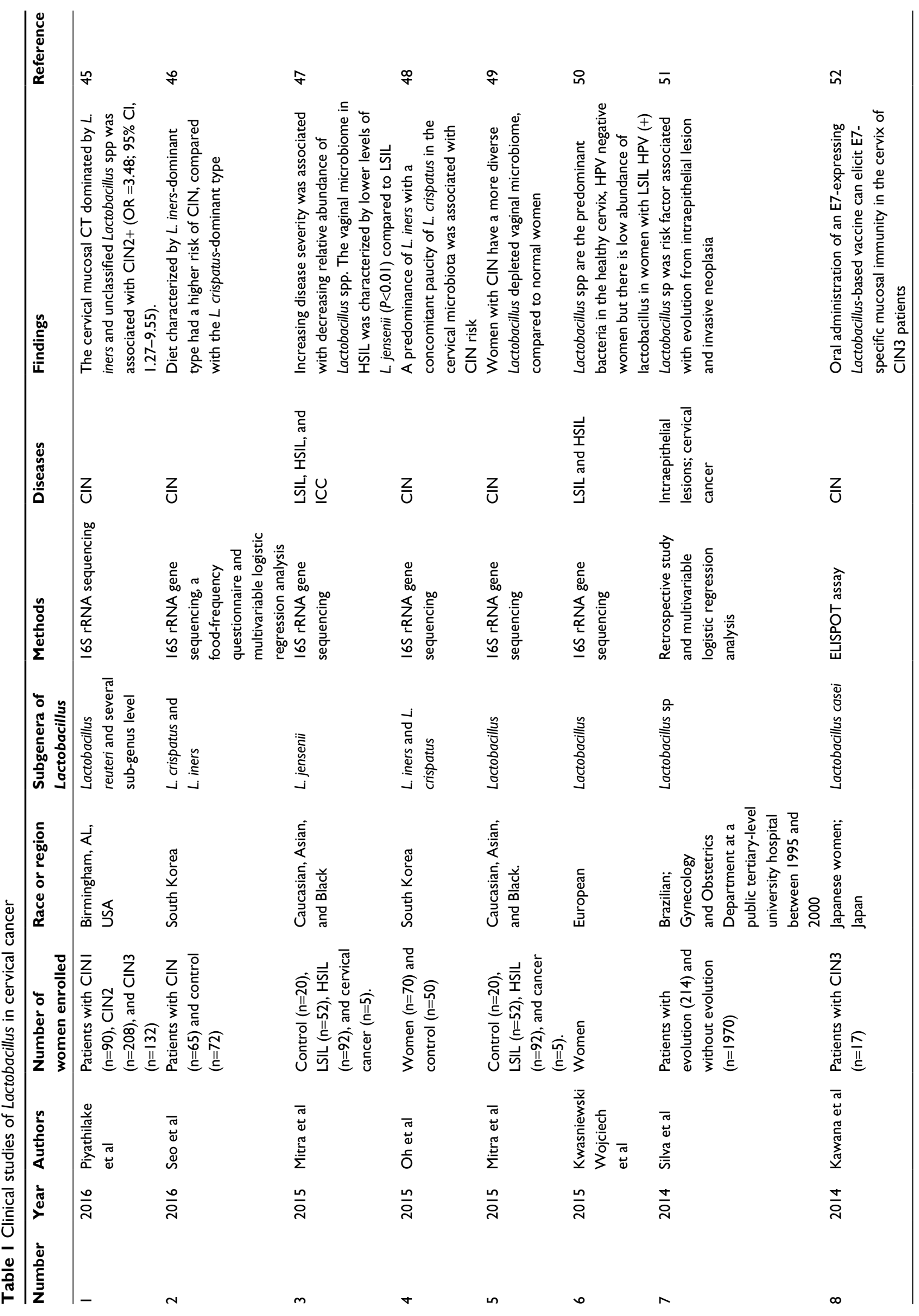




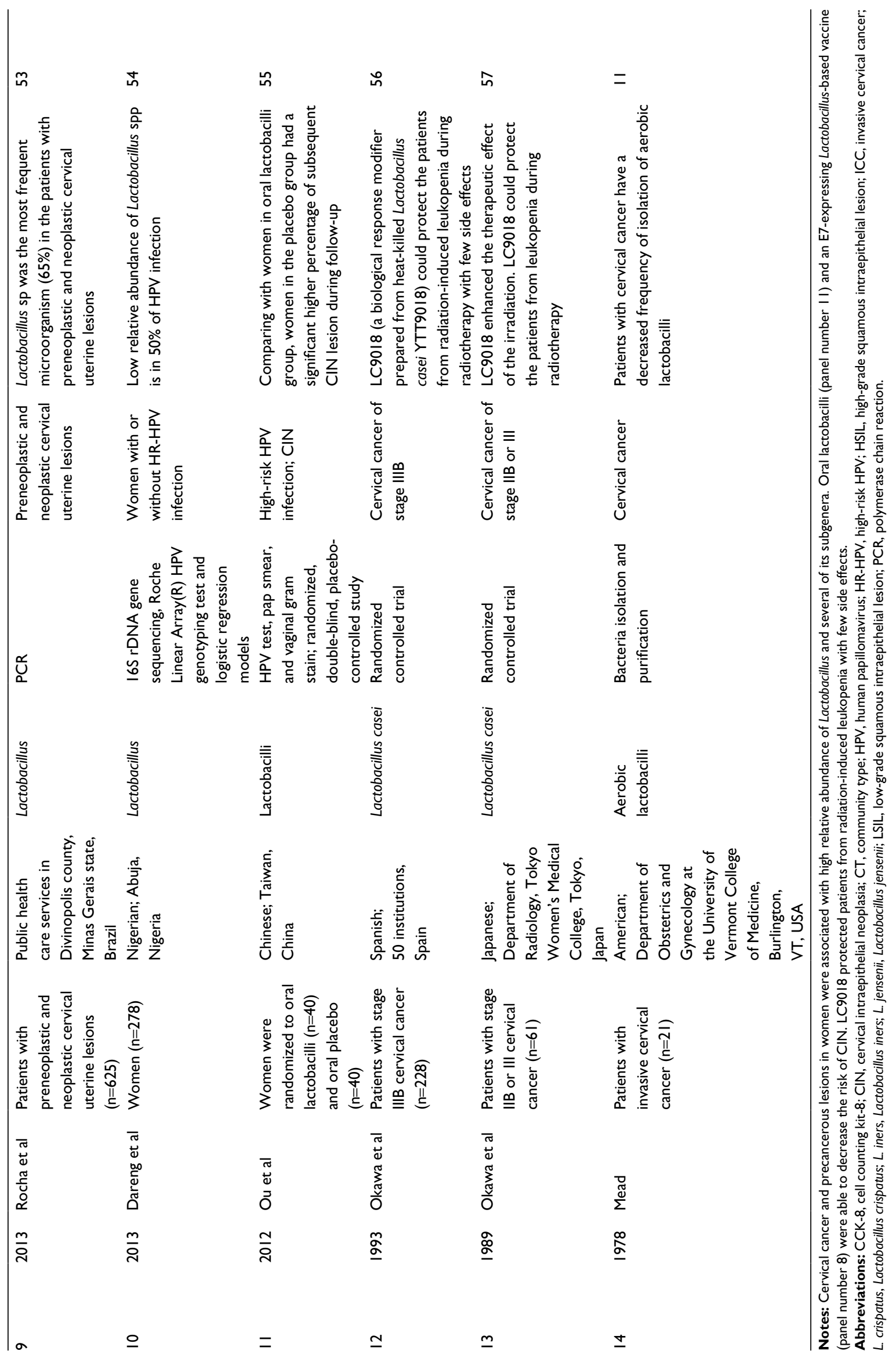




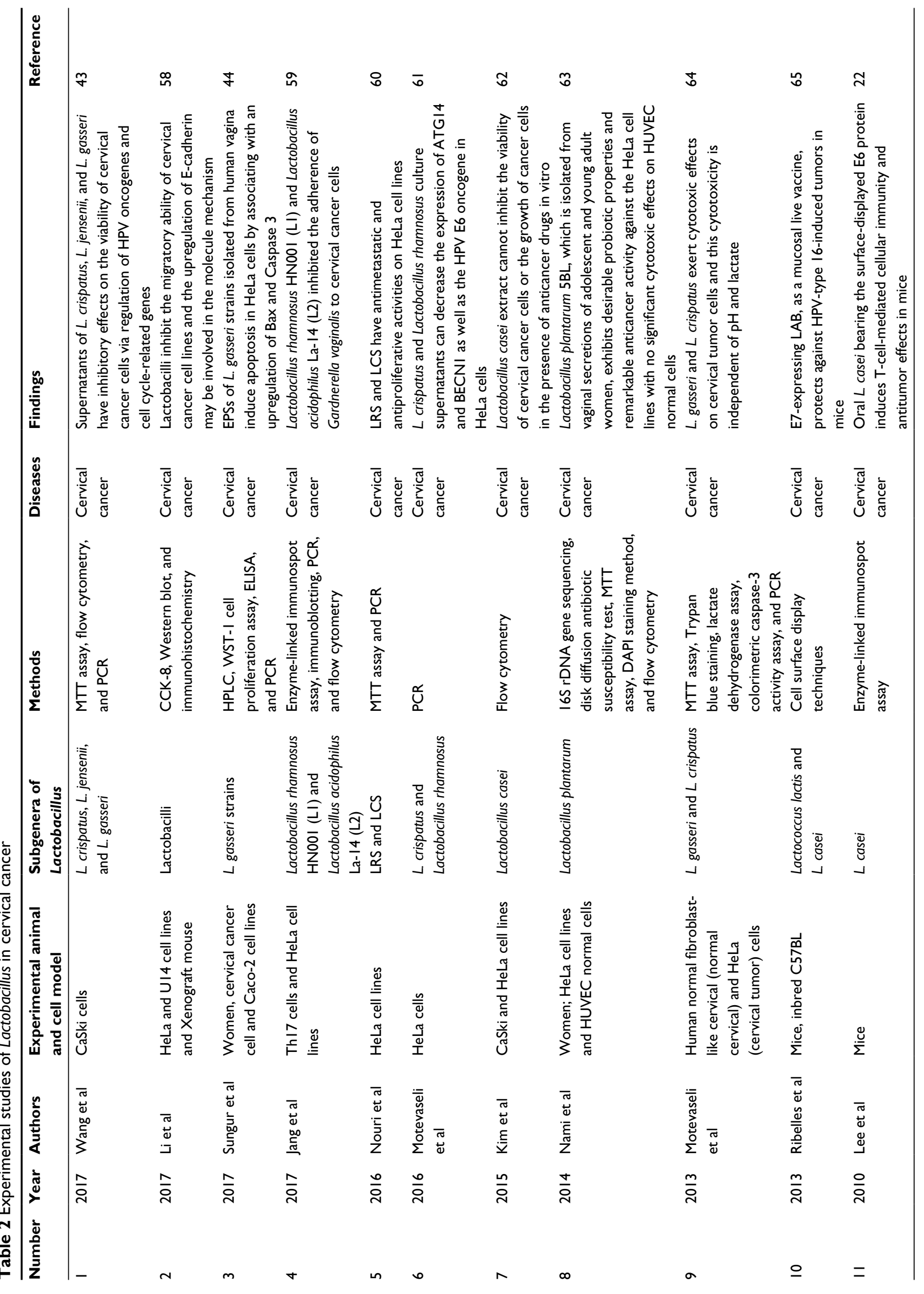




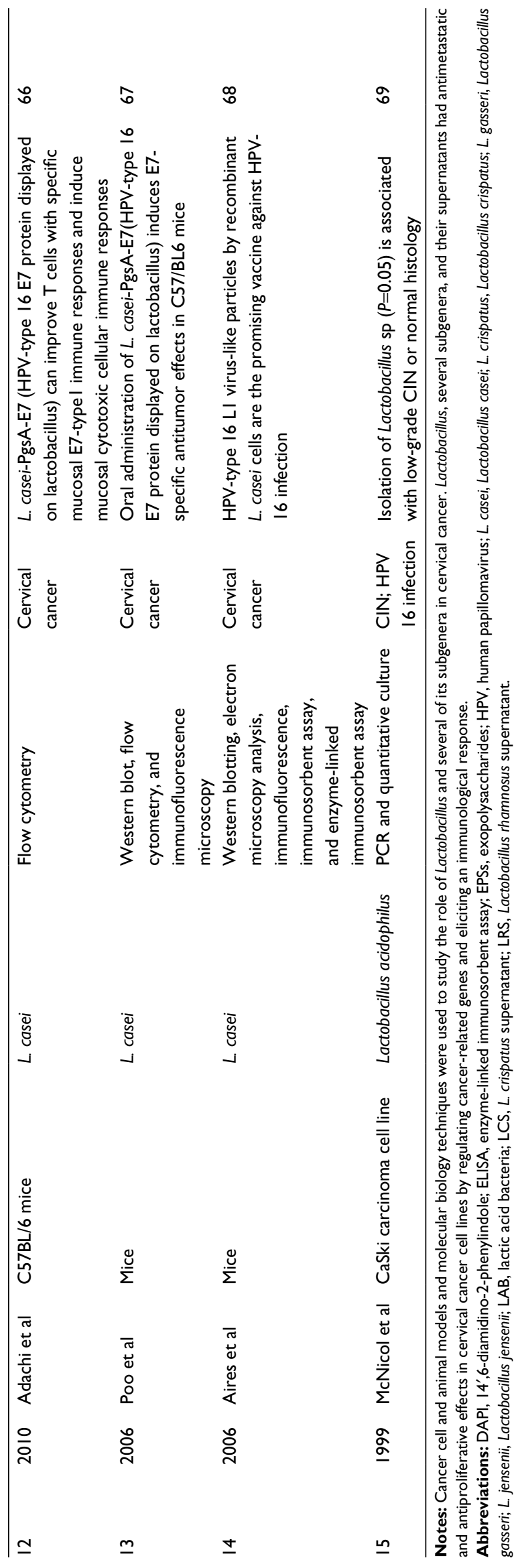

further clinical study, and clinical study can be the reliable test of experimental study. Lactobacillus as a probiotic is characterized by fewer side effects and can be used as oral preparation. The signal peptide of S-layer on monolayer cell membrane of Lactobacillus is easy to combine with the exogenous target protein. These characteristics determine the Lactobacillus as a desired vector for recombinant protein vaccine and, moreover, Lactobacillus in vaginal flora as protection against HPV-related disease. Thus, the use of Lactobacillus as a vector to construct the HPV-related protein vaccine can achieve admirable antitumor effects in clinical practice.

Many clinical studies found that a decline in the quantity and activity of Lactobacillus was involved in the initiation and progression of cervical cancer, which provides a novel insight into the use of probiotics to prevent cervical cancer. ${ }^{47,54,58}$ Some experimental studies found that Lactobacil$l u$ s and its metabolites inhibit the proliferation of cervical cancer cells by regulating cancer-related genes or through an immunological mechanism. These studies provide a theoretical basis for further clinical application of Lactobacillus in cervical cancer.

Lactobacillus and its metabolites, which are used as treatments as well as prophylactic agents, have unique advantages. Lactobacillus as a vaginal parasitical bacterium has a lower toxicity and fewer side effects compared to other bacteria. It acts mainly by regulating the microecological environment and, compared to other bacteria, is less susceptible to resistance.$^{30}$ Lactobacillus and its metabolites are efficacious and superior in clinical applications for the prevention of cervical cancer. ${ }^{31}$

In addition to cervical cancer, Lactobacillus is associated with the proliferation and regulation of cells of other cancer types, such as breast cancer, ${ }^{32}$ colorectal cancer, ${ }^{33}$ gastric cancer, ${ }^{34}$ and oral cancer. ${ }^{35,36}$ Observations in these other cancers may offer insights into the study of Lactobacillus in cervical cancer. The main observations regarding Lactobacillus in cancers are as follows: 1) Lactobacillus can activate and strengthen the antitumor effects of immunocytes such as thymus-derived cells (T cells), natural killer (NK) cells, dendritic cells (DCs), and macrophages and immunological factors produced by immunocytes; $;^{37-39}$ 2) a large amount of unmethylated dinucleotide repeat sequences present in the nuclei of Lactobacillus can activate the innate immune response by binding to a specific receptor that exists on the surface of human cells; ${ }^{40}$ 3) Lactobacillus that is suitable for reproduction and growth in an anaerobic environment can selectively accumulate in the hypoxic zones of solid cancers; ${ }^{41}$ 


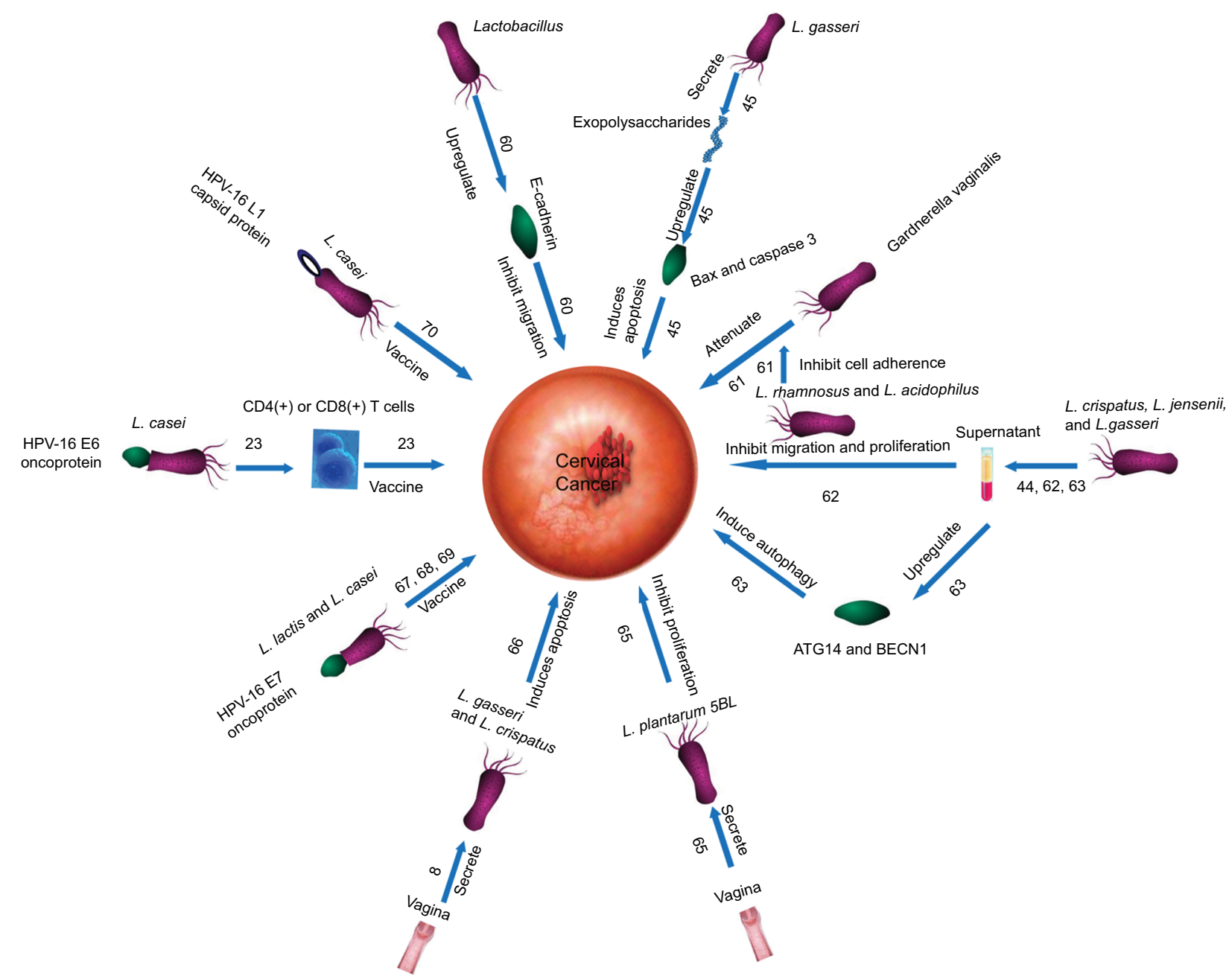

Figure 2 Network illustrating the effects of Lactobacillus in cervical cancer.

Note: An association network for Lactobacillus in cervical cancer was constructed based on the analysis of experimental studies and allows improved visualization of these studies.

Abbreviations: HPV, human papillomavirus; L. acidophilus, Lactobacillus acidophilus; L. casei, Lactobacillus casei; L. crispatus, Lactobacillus crispatus; L. gasseri, Lactobacillus gasseri; L. jensenii, Lactobacillus jensenii; L. lactis, Lactobacillus lactis; L. plantarum, Lactobacillus plantarum; L. rhamnosus, Lactobacillus rhamnosus.

and 4) Lactobacillus can selectively locate to solid cancers and can be used as a vector for gene therapy and targeted therapies. $^{35}$

The diagnosis and treatment of cervical cancer have been advanced considerably in recent years, but there is still a long way to go in regard to cancer prevention. This review cannot include some unpublished research or ongoing studies. There is also a lack of large-scale multicenter clinical trials and clinical prospective cohort studies in the literature. A perfect theory cannot be constructed for the mechanism of action of Lactobacillus in cervical cancer. Vaginal Lactobacillus and its metabolites may provide a novel insight into the prevention of cervical cancer. Thus, we put forth some future directions for further study.

\section{Future directions}

\section{Elucidate the role of vaginal Lactobacillus in cervical cancer}

Lactobacillus is the dominant bacteria in the vagina and affects the growth of other bacteria. ${ }^{42}$ There appears to be a complex relationship between cervical cancer and vaginal Lactobacillus. However, many problems deserve further consideration. How do the community structure and diversity of Lactobacillus vary with differences in race, region, lifestyle, and diet? How does Lactobacillus drive the occurrence and development of cervical cancer? What is the role of Lactobacillus metabolites in balancing vaginal microecology and the development of cervical cancer? 


\section{Elucidate the immunological functions mediated by vaginal Lactobacillus in cervical cancer}

Immunotherapy provides a broad perspective for the treatment of cancers. Vaginal Lactobacillus and its metabolites do affect the immune system in cervical cancer. ${ }^{22}$ Though much effort has been made into understanding the mechanisms of this effect, many bottlenecks must be addressed before stepping from the imbalanced vaginal microecological system to the immune system to cervical cancer genesis and development. Lactobacillus is an easily available probiotic that is safe and has no side effects or toxicity. The envelope of Lactobacillus is often used as a carrier to express an alternative antigen for vaccines, which may provide a novel idea for the primary prevention of cervical cancer. A cervical cancer vaccine based on Lactobacillus with an HPV vaccine promises to be a new method for the prevention of cervical cancer. Prospective studies on cervical cancer incidence after intervention with the Lactobacillus vaccine should be performed.

\section{Elucidate the molecular mechanism by which Lactobacillus inhibits cervical cancer}

Lactobacillus inhibits the proliferation of cells from multiple cancer types. Most studies have focused on the relationship between Lactobacillus and cancer, but the mechanisms underlying this relationship have yet to be clarified. Many enzymes and peptides and the lactic acid secreted by Lactobacillus are involved in activating and regulating important signaling molecules and pathways in cervical cancer. ${ }^{43,44}$ It is necessary to elucidate the specific molecular mechanism or construct the molecular regulatory network in future research. Lactobacillus acts as a valuable cloning vector and is currently mainly used as a plasmid vector for the experimental research of cancer in vitro. This use may suggest additional therapeutic use as a carrier to express antioncogenes or encapsulated anticarcinogens in the future.

In light of the importance of Lactobacillus in cervical cancer, much attention has been paid to the study of vaginal microecology in recent years. Lactobacillus has shown tremendous promise for the prevention and treatment of cervical cancer. However, knowledge of vaginal microecology and Lactobacillus is far from complete. More research will be required before clinical application of Lactobacillus in cervical cancer is achieved.

\section{Acknowledgment}

This work was supported by the Huzhou Public Welfare Technology Application Research Program (2016GYB14).

\section{Disclosure}

The authors report no conflicts of interest in this work.

\section{References}

1. Lv L, Wang X. MicroRNA-296 targets specificity protein 1 to suppress cell proliferation and invasion in cervical cancer. Oncol Res. Epub 2017 Dec 14.

2. Yang PM, Chou CJ, Tseng SH, Hung CF. Bioinformatics and in vitro experimental analyses identify the selective therapeutic potential of interferon gamma and apigenin against cervical squamous cell carcinoma and adenocarcinoma. Oncotarget. 2017;8(28):46145-46162.

3. Musa J, Achenbach CJ, O’Dwyer LC, et al. Effect of cervical cancer education and provider recommendation for screening on screening rates: a systematic review and meta-analysis. PLoS One. 2017;12(9):e0183924.

4. Yang J, Nolte FS, Chajewski OS, et al. Cytology and high risk HPV testing in cervical cancer screening program: outcome of 3-year followup in an academic institute. Diagn Cytopathol. 2017;46(1):22-27.

5. Chase D, Goulder A, Zenhausern F, Monk B, Herbst-Kralovetz M The vaginal and gastrointestinal microbiomes in gynecologic cancers: a review of applications in etiology, symptoms and treatment. Gynecol Oncol. 2015;138(1):190-200.

6. Hillemanns P, Soergel P, Hertel H, Jentschke M. Epidemiology and early detection of cervical cancer. Oncol Res Treat. 2016;39(9):501-506.

7. Yue XA, Chen P, Tang Y, Wu X, Hu Z. The dynamic changes of vaginal microecosystem in patients with recurrent vulvovaginal candidiasis: a retrospective study of 800 patients. Arch Gynecol Obstet. 2015;292(6):1285-1294.

8. Grmek KI, Dermota U, Golle A. Frequency of detection of Gardnerella vaginalis in vaginal smears in the upper Carniola region. Acta Dermatovenerol Alp Pannonica Adriat. 2016;25(2):31-33.

9. Sierra LJ, Brown AG, Barilá GO, et al. Colonization of the cervicovaginal space with Gardnerella vaginalis leads to local inflammation and cervical remodeling in pregnant mice. PLoS One. 2018;13(1):e0191524.

10. Adebamowo SN, Ma B, Zella D, et al; ACCME Research Group. Mycoplasma hominis and Mycoplasma genitalium in the vaginal microbiota and persistent high-risk human papillomavirus infection. Front Public Health. 2017;5:140.

11. Mead PB. Cervical-vaginal flora of women with invasive cervical cancer. Obstet Gynecol. 1978;52(5):601-604.

12. Vaneechoutte M. Lactobacillus iners, the unusual suspect. Res Microbiol. 2017;168(9-10):826-836.

13. Smith WL, Hedges SR, Mordechai E, et al. Cervical and vaginal flora specimens are highly concordant with respect to bacterial vaginosisassociated organisms and commensal Lactobacillus species in women of reproductive age. J Clin Microbiol. 2014;52(8):3078-3081.

14. Nasioudis D, Forney LJ, Schneider GM, et al. The composition of the vaginal microbiome in first trimester pregnant women influences the level of autophagy and stress in vaginal epithelial cells. $J$ Reprod Immunol. 2017;123:35-39.

15. Niu XX, Li T, Zhang X, Wang SX, Liu ZH. Lactobacillus crispatus modulates vaginal epithelial cell innate response to Candida albicans. Chin Med J (Engl). 2017;130(3):273-279.

16. Younes JA, Klappe K, Kok JW, Busscher HJ, Reid G, van der Mei HC. Vaginal epithelial cells regulate membrane adhesiveness to coordinate bacterial adhesion. Cell Microbiol. 2015;18(4):605-614.

17. Medina-Colorado AA, Vincent KL, Miller AL, et al. Vaginal ecosystem modeling of growth patterns of anaerobic bacteria in microaerophilic conditions. Anaerobe. 2017;45:10-18. 
18. Sgibnev AV, Kremleva EA. Vaginal protection by $\mathrm{H}_{2} \mathrm{O}_{2}$-producing lactobacilli. Jundishapur J Microbiol. 2015;8(10):e22913.

19. Zadravec P, Štrukelj B, Berlec A. Improvement of LysM-mediated surface display of designed ankyrin repeat proteins (DARPins) in recombinant and nonrecombinant strains of Lactococcus lactis and Lactobacillus species. Appl Environ Microbiol. 2015;81(6):2098-2106.

20. Homburg C, Bommer M, Wuttge S, et al. Inducer exclusion in Firmicutes: insights into the regulation of a carbohydrate ATP binding cassette transporter from Lactobacillus casei BL23 by the signal transducing protein P-Ser46-HPr. Mol Microbiol. 2017;105(1):25-45.

21. Wang S, Wang Q, Yang E, Yan L, Li T, Zhuang H. Antimicrobial compounds produced by vaginal Lactobacillus crispatus are able to strongly inhibit Candida albicans growth, hyphal formation and regulate virulence-related gene expressions. Front Microbiol. 2017;8:564.

22. Lee TY, Kim YH, Lee KS, et al. Human papillomavirus type 16 E6-specific antitumor immunity is induced by oral administration of HPV16 E6-expressing Lactobacillus casei in C57BL/6 mice. Cancer Immunol Immunother. 2010;59(11):1727-1737.

23. Yao XY, Yuan MM, Li DJ. Molecular adjuvant $\mathrm{C} 3 \mathrm{~d} 3$ improved the antihCGbeta humoral immune response in vaginal inoculation with live recombinant Lactobacillus expressing hCGbeta-C3d3 fusion protein. Vaccine. 2007;25(32):6129-6139.

24. Sandes S, Alvim L, Silva B, et al. Selection of new lactic acid bacteria strains bearing probiotic features from mucosal microbiota of healthy calves: looking for immunobiotics through in vitro and in vivo approaches for immunoprophylaxis applications. Microbiol Res. 2017;200(1):1-13.

25. Atkins D, Best D, Briss PA, et al. Grading quality of evidence and strength of recommendations. BMJ. 2004;328(7454):1490.

26. Wilting SM, Steenbergen RDM. Molecular events leading to HPVinduced high grade neoplasia. Papillomavirus Res. 2016;2:85-88.

27. Overman BA. The vagina as an ecologic system. Current understanding and clinical applications. J Nurse Midwifery. 1993;38(3):146-151.

28. Di Paola M, Sani C, Clemente AM, et al. Characterization of cervicovaginal microbiota in women developing persistent high-risk human papillomavirus infection. Sci Rep. 2017;7(1):10200.

29. Sowjanya AP, Rao M, Vedantham H, et al. Correlation of plasma nitrite/nitrate levels and inducible nitric oxide gene expression among women with cervical abnormalities and cancer. Nitric Oxide. 2016;52: $21-28$.

30. Collins SL, McMillan AM, Seney S, et al. Evaluation of lactitol, lactulose, raffinose, and oligofructose for maintenance of a Lactobacillus-dominated vaginal microbiota establishes a promising prebiotic candidate. Appl Environ Microbiol. 2017;84(8):AEM.02200-AEM.02217.

31. Champer M, Wong AM, Champer J, et al. The role of the vaginal microbiome in gynaecological cancer. BJOG. 2017;125(3): 309-315.

32. Marschalek J, Farr A, Marschalek ML, et al. Influence of orally administered probiotic Lactobacillus strains on vaginal microbiota in women with breast cancer during chemotherapy: a randomized placebo-controlled double-blinded pilot study. Breast Care (Basel). 2017;12(5):335-339.

33. Jacouton E, Chain F, Sokol H, Langella P, Bermúdez-Humarán LG. Probiotic strain Lactobacillus casei BL23 prevents colitis-associated colorectal cancer. Front Immunol. 2017;8:1553.

34. Rasouli BS, Ghadimi-Darsajini A, Nekouian R, Iragian GR. In vitro activity of probiotic Lactobacillus reuteri against gastric cancer progression by downregulation of urokinase plasminogen activator/urokinase plasminogen activator receptor gene expression. J Cancer Res Ther. 2017;13(2):246-251.

35. Asoudeh-Fard A, Barzegari A, Dehnad A, Bastani S, Golchin A, Omidi Y. Lactobacillus plantarum induces apoptosis in oral cancer KB cells through upregulation of PTEN and downregulation of MAPK signalling pathways. Bioimpacts. 2017;7(3):193-198.

36. Cheng Z, Xu H, Wang X, Liu Z. Lactobacillus raises in vitro anticancer effect of geniposide in HSC-3 human oral squamous cell carcinoma cells. Exp Ther Med. 2017;14(5):4586-4594.
37. Klimek R, Klimek M, Jasiczek D. Immunotherapy of cervical cancer as a biological dissipative structure. Neuro Endocrinol Lett. 2011;32(4):380-388.

38. Mohamadzadeh M, Klaenhammer TR. Specific Lactobacillus species differentially activate toll-like receptors and downstream signals in dendritic cells. Expert Rev Vaccines. 2008;7(8):1155-1164.

39. Takeda K, Okumura K. Effects of a fermented milk drink containing Lactobacillus casei strain Shirota on the human NK-cell activity. J Nutr. 2007;137(2):791S-793S.

40. Kitazawa H, Watanabe H, Shimosato T, Kawai Y, Itoh T, Saito T. Immunostimulatory oligonucleotide, CpG-like motif exists in Lactobacillus delbrueckii ssp. bulgaricus NIAI B6. Int J Food Microbiol. 2003;85(1):11-21.

41. Esfandiary A, Taherian-Esfahani Z, Abedin-Do A, et al. Lactobacilli modulate hypoxia-inducible factor (HIF)-1 regulatory pathway in triple negative breast cancer cell line. Cell J. 2016;18(2):237-244.

42. Kovachev S. Defence factors of vaginal lactobacilli. Crit Rev Microbiol. 2017:44(1):31-39.

43. Wang KD, Xu DJ, Wang BY, Yan DH, Lv Z, Su JR. Inhibitory effect of vaginal Lactobacillus supernatants on cervical cancer cells. Probiotics Antimicrob Proteins. Epub 2017 Oct 25;(suppl 1):1-7.

44. Sungur T, Aslim B, Karaaslan C, Aktas B. Impact of exopolysaccharides (EPSs) of Lactobacillus gasseri strains isolated from human vagina on cervical tumor cells (HeLa). Anaerobe. 2017;47:137-144.

45. Piyathilake CJ, Ollberding NJ, Kumar R, Macaluso M, Alvarez RD, Morrow CD. Cervical microbiota associated with higher grade cervical intraepithelial neoplasia in women infected with high-risk human papillomaviruses. Cancer Prev Res (Phila). 2016;9(5):357-366.

46. Seo SS, Oh HY, Lee JK, Kong JS, Lee DO, Kim MK. Combined effect of diet and cervical microbiome on the risk of cervical intraepithelial neoplasia. Clin Nutr. 2016;35(6):1434-1441.

47. Mitra A, MacIntyre DA, Lee YS, et al. Cervical intraepithelial neoplasia disease progression is associated with increased vaginal microbiome diversity. Sci Rep. 2015;5:16865.

48. Oh HY, Kim BS, Seo SS, et al. The association of uterine cervical microbiota with an increased risk for cervical intraepithelial neoplasia in Korea. Clin Microbiol Infect. 2015;21(7):674.e1-e9.

49. Mitra A, MacIntyre DA, Lee YS, et al. The vaginal microbiome of women with cervical intraepithelial neoplasia. Microbiome. 2015;9(25):976-977.

50. Wojciech K, Kotarski J, Barczynski B, et al. A meta genomic approach to characterization of the cervix microbiome during HPV dependent carcinogenesis. Int J Gynecol Cancer. 2015;9(25):848.

51. Silva C, Almeida EC, Côbo Ede C, Zeferino VF, Murta EF, Etchebehere RM. A retrospective study on cervical intraepithelial lesions of lowgrade and undetermined significance: evolution, associated factors and cytohistological correlation. Sao Paulo Med J. 2014;132(2):92-96.

52. Kawana K, Adachi K, Kojima S, et al. Oral vaccination against HPV E7 for treatment of cervical intraepithelial neoplasia grade 3 (CIN3) elicits E7-specific mucosal immunity in the cervix of CIN3 patients. Vaccine. 2014;32(47):6233-6239.

53. Rocha VR, Schüffner Rde O, Soares II, et al. Cervical uterine lesions: an epidemiological and molecular investigation in Midwestern Minas Gerais, Brazil. J Med Virol. 2013;85(5):860-865.

54. Dareng EO, Ma B, Famooto AO, et al. Prevalent high-risk HPV infection and vaginal microbiota in Nigerian women. Epidemiol Infect. 2016;144(1):123-137.

55. Ou YC, Lin H Randomized, double-blind, placebo-controlled study of oral lactobacilli to facilitate the clearance of genital high risk HPV infection in women. Int J Gynecol Cancer. 2012;141(22):E695.

56. Okawa T, Niibe H, Arai T, et al. Effect of LC 9018 combined with radiation therapy on carcinoma of the uterine cervix. A phase III, multicenter, randomized, controlled study. Cancer. 1993;72(6):1949-1954.

57. Okawa T, Kita M, Arai T, et al. Phase II randomized clinical trial of LC9018 concurrently used with radiation in the treatment of carcinoma of the uterine cervix. Its effect on tumor reduction and histology. Cancer. 1989;64(9):1769-1776. 
58. Li X, Wang H, Du X, et al. Lactobacilli inhibit cervical cancer cell migration in vitro and reduce tumor burden in vivo through upregulation of E-cadherin. Oncol Rep. 2017;38(3):1561-1568.

59. Jang SE, Jeong JJ, Choi SY, Kim H, Han MJ, Kim DH. Lactobacillus rhamnosus HN001 and Lactobacillus acidophilus La-14 attenuate Gardnerella vaginalis-infected bacterial vaginosis in mice. Nutrients. 2017;9(6):E531.

60. Nouri Z, Karami F, Neyazi N, et al. Dual anti-metastatic and antiproliferative activity assessment of two probiotics on HeLa and HT-29 cell lines. Cell J. 2016;18(2):127-134.

61. Motevaseli E, Azam R, Akrami SM, et al. The effect of Lactobacillus crispatus and Lactobacillus rhamnosus culture supernatants on expression of autophagy genes and HPV E6 and E7 oncogenes in the HeLa cell line. Cell J. 2016;17(4):601-607.

62. Kim SN, Lee WM, Park KS, Kim JB, Han DJ, Bae J. The effect of Lactobacillus casei extract on cervical cancer cell lines. Contemp Oncol (Pozn). 2015;19(4):306-312.

63. Nami Y, Abdullah N, Haghshenas B, Radiah D, Rosli R, Khosroushahi AY. Assessment of probiotic potential and anticancer activity of newly isolated vaginal bacterium Lactobacillus plantarum 5BL. Microbiol Immunol. 2014;58(9):492-502.
64. Motevaseli E, Shirzad M, Akrami SM, Mousavi AS, Mirsalehian A, Modarressi MH. Normal and tumour cervical cells respond differently to vaginal lactobacilli, independent of $\mathrm{pH}$ and lactate. J Med Microbiol. 2013;62(pt 7):1065-1072.

65. Ribelles P, Benbouziane B, Langella P, Suárez JE, Bermúdez-Humarán LG. Protection against human papillomavirus type 16-induced tumors in mice using non-genetically modified lactic acid bacteria displaying E7 antigen at its surface. Appl Microbiol Biotechnol. 2013;97(3):1231-1239.

66. Adachi K, Kawana K, Yokoyama T, et al. Oral immunization with a Lactobacillus casei vaccine expressing human papillomavirus (HPV) type 16 E7 is an effective strategy to induce mucosal cytotoxic lymphocytes against HPV16 E7. Vaccine. 2010;28(16):2810-2817.

67. Poo H, Pyo HM, Lee TY, et al. Oral administration of human papillomavirus type 16 E7 displayed on Lactobacillus casei induces E7-specific antitumor effects in C57/BL6 mice. Int J Cancer. 2006;119(7):1702-1709.

68. Aires KA, Cianciarullo AM, Carneiro SM, et al. Production of human papillomavirus type $16 \mathrm{~L} 1$ virus-like particles by recombinant Lactobacillus casei cells. Appl Environ Microbiol. 2006;72(1):745-752.

69. McNicol PJ, Paraskevas M, Guijon FB. The effect of vaginal microbes on in vivo and in vitro expression of human papillomavirus 16 E6-E7 genes. Cancer Detect Prev. 1999;23(1):13-21.
Cancer Management and Research

\section{Publish your work in this journal}

Cancer Management and Research is an international, peer-reviewed open access journal focusing on cancer research and the optimal use of preventative and integrated treatment interventions to achieve improved outcomes, enhanced survival and quality of life for the cancer patient The manuscript management system is completely online and includes

\section{Dovepress}

a very quick and fair peer-review system, which is all easy to use. Visit http://www.dovepress.com/testimonials.php to read real quotes from published authors. 